\title{
Entanglement versus Bell violations and their behaviour under local filtering operations
}

\author{
Frank Verstraetef \\ Department of Mathematical Physics and Astronomy, Ghent University, Belgium \\ Department of Electrical Engineering (SISTA), KULeuven, Belgium \\ Michael M. Wolff \\ Institute for Mathematical Physics, TU Braunschweig, Germany
}

(Dated: November 2, 2018)

\begin{abstract}
We discuss the relations between the violation of the CHSH Bell inequality for systems of two qubits on the one side and entanglement of formation, local filtering operations, and the entropy and purity on the other. We calculate the extremal Bell violations for a given amount of entanglement of formation and characterize the respective states, which turn out to have extremal properties also with respect to the entropy, purity and several entanglement monotones. The optimal local filtering operations leading to the maximal Bell violation for a given state are provided and the special role of the resulting Bell diagonal states in the context of Bell inequalities is discussed.
\end{abstract}

\section{INTRODUCTION AND PRELIMINARIES}

Entanglement has always been a key issue in the ongoing debate about the foundations and interpretation of quantum mechanics since Einstein, Podolsky and Rosen (EPR) published their famous gedanken-experiment in 1935 11. For a long time discussions about entanglement were purely meta-theoretical. However, this appeal was changed dramatically in 1964 by John Bell's [2] observation that the EPR dilemma could be formulated in the form of assumptions naturally leading to a falsifiable prediction. The experimental fact that these Bell inequalities can indeed be violated [3] has not only ruled out a single theory, but the very way theories had been formulated for quite a long time. Whereas until 1989 entanglement was widely believed to be equivalent to the violation of a Bell inequality, it turned out that such a violation is neither necessary for mixed entangled states [4] nor a good measure for the amount of entanglement [5, 6. It was in particular shown by Gisin [6] that some states initially satisfying Bell's inequalities, lead to a violation after certain local filtering operations. Hence, local filtering operations can on an average increase the degree of violation while decreasing the amount of entanglement. Although general structural knowledge about entanglement [7] on the one side and Bell's inequalities [8] on the other has increased dramatically in the last few years, our knowledge about their relation is still mainly restricted to the fact that states violating a Bell inequality have to be entangled.

The present paper is devoted to settling the relationship between entanglement, measured in terms of the concurrence, the Bell violations and their behaviour under local filtering operations for the case of two qubit systems.

To fix ideas we will start by recalling some of the basic definitions and properties. Throughout this paper we will consider systems of two qubits - one may think of two spin $\frac{1}{2}$ particles or the polarization degrees of freedom of two photons - for which we can explicitly calculate the amount of entanglement, in terms of the entanglement of formation [9], as well as the maximal violation of the Bell inequality in its Clauser-Horne-Shimony-Holt (CHSH) form 10, 11].

The concept of entanglement of formation $(E o F)$ is related to the amount of entanglement needed to prepare the state $\rho$, and it was shown by Wootters [9] that

$$
\operatorname{EoF}(\rho)=h\left(\frac{1+\sqrt{1-C^{2}}}{2}\right),
$$

where $h(x)=-x \lg x-(1-x) \lg (1-x)$ and the concurrence $C=\max \left[0, \sqrt{l_{1}}-\sum_{i=2}^{4} \sqrt{l_{i}}\right]$ with $\left\{l_{i}\right\}$ being the decreasingly ordered eigenvalues of $\rho\left(\sigma_{y} \otimes \sigma_{y}\right) \rho^{T}\left(\sigma_{y} \otimes \sigma_{y}\right)$. In order to circumvent lengthy logarithmic expressions we will in following use the concurrence rather than $E o F$, which is in fact a convex and monotone function with respect to $C$.

The CHSH inequality formulated for two qubit systems states, that within any local classical model the expectation value $\operatorname{Tr}(\rho \mathcal{B})$ of the Bell operator

$$
\mathcal{B}=\frac{1}{2} \sum_{i j=1}^{3}\left[a_{i}\left(c_{j}+d_{j}\right)+b_{i}\left(c_{j}-d_{j}\right)\right] \sigma_{i} \otimes \sigma_{j},
$$

where $(\vec{a}, \vec{b}, \vec{c}, \vec{d})$ are real unit vectors and $\sigma_{i}$ being the Pauli matrices, has to be bounded by one. Its violation is a measure of how strong non-classical properties of the state manifest themselves in correlation experiments.

In the sequel we will often represent the two qubit state in terms of the $4 \times 4$ matrix $\tilde{R}_{i j}=\operatorname{Tr}\left(\rho \sigma_{i} \otimes \sigma_{j}\right)$ (with $\sigma_{0}$ being the identity) and the $3 \times 3$ block $R_{k l}=$ $\operatorname{Tr}\left(\rho \sigma_{k} \otimes \sigma_{l}\right)$, where $k, l=1,2,3$. It is important to note, that the latter can be diagonalized just by changing the local bases, which will neither affect the entanglement nor the maximal Bell violation. 


\section{ENTANGLEMENT OF FORMATION VERSUS VIOLATION OF THE CHSH INEQUALITY}

In [11] the Horodecki family showed, that the maximal violation of the CHSH inequality can be calculated by considering the $3 \times 3$ matrix $R_{k l}=\operatorname{Tr}\left(\rho \sigma_{k} \otimes \sigma_{l}\right)$. We will give an alternative derivation of this result in a way that will be very useful in the sequel:

Lemma 1 (Horodecki [11]) Given the decreasingly ordered singular values $\left\{\sigma_{i}\right\}$ of $R$, the maximal violation $\beta(\rho)=\max _{\mathcal{B}} \operatorname{Tr}(\rho \mathcal{B})$ is given by $\sqrt{\sigma_{1}^{2}+\sigma_{2}^{2}}$.

Proof: Translated into the $R$-picture, calculating the maximal expectation value of $\mathcal{B}$ under the constraint that $(\vec{a}, \vec{b}, \vec{c}, \vec{d})$ are real unit vectors, amounts to maximizing $\operatorname{Tr}(R X)$ with

$$
X=\left(\begin{array}{ll}
\vec{c} & \vec{d}
\end{array}\right) \frac{1}{2}\left(\begin{array}{cc}
1 & 1 \\
1 & -1
\end{array}\right)\left(\begin{array}{l}
\vec{a}^{T} \\
\vec{b}^{T}
\end{array}\right) .
$$

It is an easy exercise to show that $X$ is a real $3 \times 3$ matrix, subjected to the only constraints that it be of rank 2 and that $\operatorname{Tr}\left(X^{T} X\right)=1$. Standard linear algebra then dictates that $\operatorname{Tr}(R X)$ is maximized iff $X$ is chosen to be proportional to the best rank 2 least-squares approximation of the matrix $R$. In the basis where $R$ is diagonal $\left(R=\operatorname{diag}\left(\sigma_{1}, \sigma_{2}, \sigma_{3}\right)\right), X$ is therefore given by $X=\operatorname{diag}\left(\sigma_{1}, \sigma_{2}, 0\right) / \sqrt{\sigma_{1}^{2}+\sigma_{2}^{2}}$, which immediately leads to $\beta=\sqrt{\sigma_{1}^{2}+\sigma_{2}^{2}}$.

In the following we will derive the extremal violations for a given amount of entanglement plotted in Fig.1].

Theorem 1 The maximal violation of the $C H S H$ inequality for given concurrence $C$ is $\beta(\rho)=\sqrt{1+C^{2}(\rho)}$.

Proof: As shown by Wootters [9], it is possible to decompose a mixed state of two qubits $\rho=\sum_{i} p_{i}\left|\psi_{i}\right\rangle\left\langle\psi_{i}\right|$ into a convex sum of pure states, all with concurrence equal to the concurrence of the mixed state. Since the extremal violation is moreover a convex function, i.e., $\max _{\mathcal{B}} \operatorname{Tr}(\rho \mathcal{B}) \leq \sum_{i} p_{i} \max _{\mathcal{B}}\left\langle\psi_{i}|\mathcal{B}| \psi_{i}\right\rangle$, it is sufficient to have a look at pure states, which can always be written in their Schmidt form as $|\psi\rangle=\lambda_{+}|00\rangle+\lambda_{-}|11\rangle$ with $\lambda_{ \pm}=(\sqrt{1+C} \pm \sqrt{1-C}) / 2$. The corresponding $R$-matrix is diagonal with singular values $(1, C, C)$ leading to $\beta=\sqrt{1+C^{2}}$.

It is interesting to note that there also exist mixed states of rank 2 for which the violation is as strong as for pure states. These are, up to local unitary operations, all of the form

$$
\rho=\frac{1}{2}\left(\begin{array}{cccc}
\cdot & \cdot & \cdot & \cdot \\
\cdot & 1-a & C & \cdot \\
\cdot & C & 1+a & \cdot \\
\cdot & \cdot & \cdot & \cdot
\end{array}\right)
$$

with $C$ being the concurrence and $a$ a free real parameter constrained by $|a| \leq \sqrt{1-C^{2}}$, where equality leads to

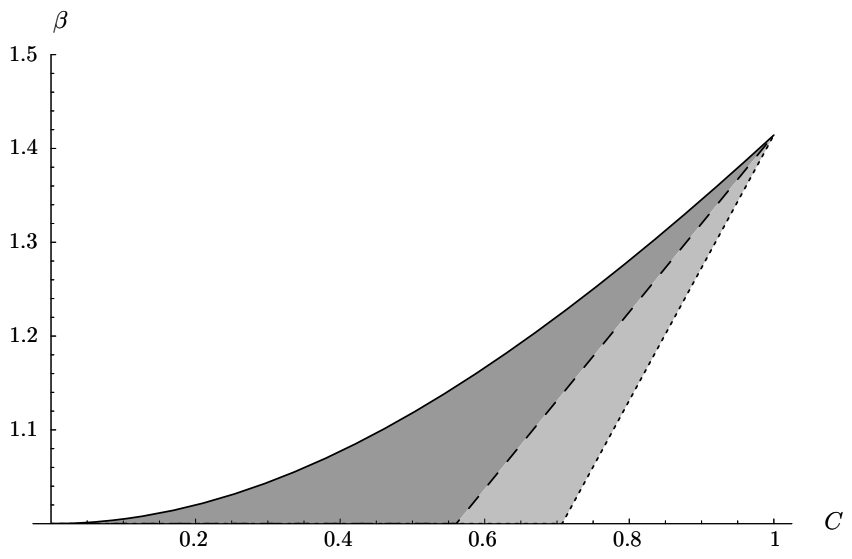

FIG. 1: The region of possible maximal Bell violation for given concurrence. The dark grey region corresponds to Bell diagonal states and the three lines represent pure states (solid), Werner states (dashed) and maximally entangled mixed states (dotted).

pure states and Bell diagonal states (see section below) are obtained for $a=0$.

Theorem 2 The minimal violation of the $C H S H$ inequality for given concurrence $C$ is given by $\beta(\rho)=$ $\max [1, \sqrt{2} C(\rho)]$.

The proof is quite technical and may be skipped by readers not interested in technical details.

Proof: We will use similar techniques as used in 12, 13, 14, where it was shown that surfaces of constant concurrence can be generated by transforming $\tilde{R} \mapsto \tilde{R}^{\prime}=$ $L_{1} \tilde{R} L_{2}^{T}$ by left and right multiplication with proper orthochronous Lorentz transformations, taken into account the constraint that the $(0,0)$ element of $\tilde{R}$ (representing the trace of $\rho$ ) does not change under these transformations. They leave the Lorentz singular values 13 invariant, and the concurrence is a function of these four parameters only.

Using the variational characterization used in lemma 1. the first step consists of varying the Lorentz transformations $L_{1}, L_{2}$ and the $3 \times 3$ rank 2 matrix $X$ (with constraint $\operatorname{Tr}\left(X^{T} X\right)=1$ ), and imposing that these variations be zero (i.e. we have an extremum). The object function is given by

$$
\operatorname{Tr}\left(L_{1} \tilde{R} L_{2}^{T}\left(\begin{array}{cc}
0 & 0 \\
0 & X
\end{array}\right)\right)
$$

under the constraints $\operatorname{Tr}\left(X^{T} X\right)=1$ and

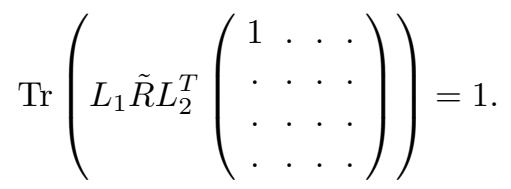

The orthogonal degrees of freedom of $X$ can be absorbed into $L_{1}, L_{2}$, such as to yield a diagonal $X$ of rank 2 : 
$X=\operatorname{diag}(q, r, 0)$ with $q^{2}+r^{2}=1$. Variation of the Lorentz transformations yields the extremal conditions

$$
\operatorname{Tr}\left(G_{1} \tilde{R}^{\prime}\left(\begin{array}{cc}
\lambda & 0 \\
0 & X
\end{array}\right)\right)=\operatorname{Tr}\left(\tilde{R}^{\prime} G_{2}\left(\begin{array}{cc}
\lambda & 0 \\
0 & X
\end{array}\right)\right)=0 .
$$

for all possible generators $G_{1}, G_{2}$ of the Lorentz group and $\lambda$ being a Lagrange parameter. The generators are all of the form

$$
G=\left(\begin{array}{cc}
0 & \vec{v} \\
\vec{v}^{T} & A
\end{array}\right)
$$

with $\vec{v} \in \mathbb{R}^{3}$ and $A$ a real and antisymmetric $3 \times 3$ block. A detailed discussion of the case $\lambda \neq 0$ shows, that this leads to the maximal violation, which we have already obtained in theorem 10 using a more simple argumentation. The minimal value of the Bell violation turns out to correspond to the case where $\lambda=0$ and yields the condition that $\tilde{R}^{\prime}$ is of form

$$
\tilde{R}^{\prime}=\left(\begin{array}{cccc}
1 & \cdot & \cdot & a \\
\cdot & x & \cdot & \cdot \\
\cdot & \cdot & y & \cdot \\
b & \cdot & \cdot & z
\end{array}\right)
$$

The extremal violation of the Bell inequality is then directly found by varying the remaining diagonal elements of $X$, leading to a violation given by $\sqrt{x^{2}+y^{2}}$. The concurrence of the extremal state can be calculated explicitly, and is given by:

$$
\begin{gathered}
C=\frac{1}{2} \max \left[0,|x-y|-\sqrt{(1-z)^{2}-(a-b)^{2}},\right. \\
\left.|x+y|-\sqrt{(1+z)^{2}-(a+b)^{2}}\right] .
\end{gathered}
$$

The constraints that $\tilde{R}$ corresponds to a (positive) state are expressed by the inequalities

$$
\begin{aligned}
-1 \leq & z \leq 1, \\
(1+z)^{2}-(a+b)^{2} & \geq(x-y)^{2}, \\
(1-z)^{2}-(a-b)^{2} & \geq(x+y)^{2} .
\end{aligned}
$$

Applying these to the expression of the concurrence, this immediately leads to the sharp inequality $C \leq$ $\min (|x|,|y|)$. The Bell violation, given by $\beta=\sqrt{x^{2}+y^{2}}$, will then be minimal for given concurrence if $|x|=|y|$, leading to final result: $\beta(\rho) \geq \sqrt{2} C(\rho)$. To complete the proof, we still have to check if there indeed exists a state with the properties that $x=y,(1+z)^{2}=(\alpha+\beta)^{2}$, $(1-z)^{2}-(\alpha-\beta)^{2} \geq(x+y)^{2},-1 \leq z \leq 1$ and $|z| \leq|x|$. Choosing for example $\alpha=\beta=(1+z) / 2$ and $z=-|x|$ indeed leads to a possible result, which is a convex combination of a maximally entangled and an orthogonal separable pure state. Note that all parameters fulfilling the above constraints lead to states with the same minimal possible amount of $\beta$ for given concurrence.
The states minimizing the Bell violation for given entanglement of formation are all rank deficient and belong to the class of maximally entangled mixed states introduced by Ishizaka and Verstraete et al. [15, 16]. These states do have a remarkable property: their entanglement of formation, negativity [17 and relative entropy of entanglement 18 cannot be increased by any global unitary transformation [16] (and thus under any transformation preserving the spectrum). For given entanglement their entropy is the largest and their purity (measured in terms of $\left.\operatorname{Tr}\left(\rho^{2}\right)\right)$ is the smallest possible one. In [12] it was shown that these states also minimize the negativity and the relative entropy of entanglement for a fixed amount of entanglement of formation, which is fully compatible with the result about the minimal violation of the Bell inequality.

\section{OPTIMAL FILTERING}

Local filtering operations on single copies are of particular importance whenever it is difficult or even impossible to operate jointly on several copies - such as in single photon experiments. Gisin [6] noted that there exist mixed states that do not violate any CHSH inequality but can violate them after a filtering operation is applied to them. Therefore the question is raised: what local filtering operation has to be applied to a given state such as to yield a new state that violates the CHSH inequality maximally?

Theorem 3 Given a single copy of a state $\rho$, then the optimal local filtering operations yielding a state with maximal possible violation of the CHSH inequality are the unique stochastically reversible filtering operations bringing the state into Bell diagonal form.

Proof: The proof is completely similar to the proof of theorem 2, so we will only repeat the major steps. In the $\tilde{R}$-picture, filtering operations correspond to left and right multiplication with Lorentz transformations, followed by renormalization. The function, which we have to maximize with respect to $L_{1}, L_{2}$ and $X=\operatorname{diag}(q, r, 0)$ in order to obtain the maximal Bell violation, therefore becomes

$$
\operatorname{Tr}\left(\frac{L_{1} \tilde{R} L_{2}^{T}}{\left(L_{1} \tilde{R} L_{2}^{T}\right)_{00}}\left(\begin{array}{cc}
0 & 0 \\
0 & X
\end{array}\right)\right)
$$

with the constraint $q^{2}+r^{2}=1$ and the normalization factor $\left(L_{1} \tilde{R} L_{2}^{T}\right)_{00}$. Variation leads to the condition

$$
\operatorname{Tr}\left(G_{1} \tilde{R}^{\prime}\left(\begin{array}{cc}
-\beta & 0 \\
0 & X
\end{array}\right)\right)=\operatorname{Tr}\left(\tilde{R}^{\prime} G_{2}\left(\begin{array}{cc}
-\beta & 0 \\
0 & X
\end{array}\right)\right)=0,
$$

where again this has to hold for arbitrary $G_{1}, G_{2}$, and where $\beta$ is equal to Eq. (14), i.e., the Bell expectation 
value for given $q, r, L_{1}, L_{2}$. If $\beta>1$ (i.e. Bell violation), it holds that $\beta$ cannot be equal to $|q|$ or $|r|$, and the form of the generators in Eq. (8) implies that the above equations can only be satisfied iff $\tilde{R}^{\prime}$ is diagonal corresponding to a Bell diagonal state (see next section). In [13 it was shown that for each mixed state there exist local filtering operations bringing the state into a unique Bell diagonal form, such that we have proven that these are the filtering operations that maximize the Bell violation. For a more detailed discussion of these filtering operations we refer to Ref. 13.

This result was expected as it was shown in 13] that exactly the same filtering operations maximize the entanglement of formation and the negativity.

Theorem 3 implies that there exists a large number of mixed entangled states that do not violate any CHSH inequality. A specific example was already given by Werner [4], and here we have shown that whatever state whose Bell diagonal normal form does not violate the $\mathrm{CHSH}$ inequalities cannot violate any $\mathrm{CHSH}$ inequality, even after all possible local filtering operations.

In the following section we will discuss in more detail the Bell diagonal states, for which theorem 3 shows that the Bell violation cannot be increased by any local filtering operation.

\section{THE ROLE OF BELL DIAGONAL STATES}

We call a state Bell diagonal if there is a local choice of bases such that it can be written as a convex combination of the four maximally entangled Bell states [20], which means that $\tilde{R}$ is diagonal in that basis. The diagonal elements of $R$ then only depend on the eigenvalues $\lambda_{1} \geq$ $\ldots \geq \lambda_{4}$ of the Bell diagonal state $[13$ and it is thus straight forward to show that the maximal Bell violation is

$$
\beta=\sqrt{2} \sqrt{\left(\lambda_{2}-\lambda_{3}\right)^{2}+\left(\lambda_{1}-\lambda_{4}\right)^{2}} .
$$

Since the concurrence is given by $C=\max \left[0,2 \lambda_{1}-1\right]$ the region of possible violations is in this case

$$
\sqrt{2}(2 C+1) / 3 \leq \beta \leq \sqrt{1+C^{2}}
$$

where the lower bound is sharp for Werner states [4] and the upper bound is attained for rank 2 Bell diagonal states and is equal to the relation for pure states.

The fact that the Bell operator $\mathcal{B}$ in Eq.(2) is itself Bell diagonal due to $\operatorname{Tr}\left(\mathcal{B} \sigma_{i} \otimes \sigma_{0}\right)=\operatorname{Tr}\left(\mathcal{B} \sigma_{0} \otimes \sigma_{i}\right)=0$ already suggests that Bell diagonal states play a special role in the context of violations of the $\mathrm{CHSH}$ inequality. And in fact, in addition to being the optimal outcomes of local filtering operations, they exhibit another special property:
Theorem 4 For any given spectrum of the density matrix the respective Bell diagonal state $\rho$ maximizes the Bell violation, i.e. $\forall U \in U(4): \beta(\rho) \geq \beta\left(U \rho U^{*}\right)$.

Proof: First note that as we have to calculate a supremum over all unitary rotations of the state $\rho$, we can without loss of generality assume that the initial state commutes with the Bell operator $\mathcal{B}$. The proof of the theorem is then based on the fact that if $u_{i k}$ are the matrix elements of a unitary matrix, then $\left|u_{i k}\right|^{2}$ is a doubly stochastic matrix, i.e., a convex combination of permutations $\tau$ (cf. 21]). If $\left\{\lambda_{i}\right\},\left\{b_{i}\right\}$ are the decreasingly ordered eigenvalues of $\rho$ resp. $\mathcal{B}$, then

$$
\begin{aligned}
\operatorname{Tr}\left(U \rho U^{*} \mathcal{B}\right) & =\sum_{i k} \lambda_{i} b_{k}\left|u_{i k}\right|^{2}=\sum_{\tau} p_{\tau} \sum_{i} \lambda_{i} b_{\tau(i)} \\
& \leq \sum_{i} \lambda_{i} b_{i}=\operatorname{Tr}(\rho \mathcal{B})
\end{aligned}
$$

This immediately implies that if we fix any spectral property of the state, such as the purity $\operatorname{Tr}\left(\rho^{2}\right)$ or the entropy $-\operatorname{Tr}(\rho \log \rho)$, the maximal violation of the $\mathrm{CHSH}$ inequality will always be attained for Bell diagonal states.

\section{CONCLUSION}

We derived the range of Bell violations for a given amount of entanglement (measured in terms of the concurrence) and discussed the extremal states, which turned out to have extremal properties also with respect to several entanglement monotones, the entropy and purity. It was conjectured by Munro et al. 22 that for given concurrence the Bell violation increases with the purity of the state. Although this is not true in general (which can already be seen from Eq.(15)), our results show that this is indeed true for the extremal cases.

Moreover, we proved that the local single copy filtering operations which maximize the concurrence and other entanglement monotones also maximize the Bell violation and lead to Bell diagonal states, which in turn are optimal with respect to global unitary operations as well.

FV acknowledges the hospitality of the TU Braunschweig, and is grateful to Jeroen Dehaene for valuable comments.

* Electronic address: frank.verstraete@rug.ac.be

$\dagger$ Electronic address: mm.wolf@tu-bs.de

[1] A. Einstein, B. Podolsky, and N. Rosen. Phys. Rev., 47:777, 1935.

[2] J.S. Bell. Physics, 1:195, 1964.

[3] A. Aspect, P. Grangier, and G. Roger. Phys. Rev. Lett., 47:460, 1981.

[4] R.F. Werner. Phys. Rev. A, 40:4277, 1989.

[5] S. Popescu. Phys. Rev. Lett., 74:2619, 1995. 
[6] N. Gisin. Phys. Lett. A, 210:151, 1996.

[7] M. Horodecki, P. Horodecki, and R. Horodecki in Quantum Information - an introduction to basic theoretical concepts and experiments, (Springer tracts in modern physics, Berlin, 2001).

[8] M. Werner and M.M. Wolf Quant. Inf. Comp., 1:1, 2001.

[9] W. Wootters. Phys. Rev. Lett., 80:2245, 1998.

[10] J.F. Clauser, M.A. Horne, A. Shimony, and R.A. Holt Phys. Rev. Lett., 23:880, 1969.

[11] R. Horodecki, P. Horodecki, and M. Horodecki Phys. Lett. A, 200:340, 1995.

[12] F. Verstraete, K. Audenaert, J. Dehaene, and B. De Moor. J. Phys. A, 34:10327, (2001).

[13] F. Verstraete, J. Dehaene, and B. De Moor. Phys. Rev. A, 64:010101(R), 2001.
[14] F. Verstraete, J. Dehaene, and B. De Moor. quant$\mathrm{ph} / 0108043$.

[15] S. Ishizaka and T. Hiroshima. Phys. Rev. A, 62:22310, 2000.

[16] F. Verstraete, K. Audenaert, and B. De Moor. Phys. Rev. A, 64:012316, 2001.

[17] G. Vidal and R.F. Werner. quant-ph/0102117.

[18] V. Vedral and M. Plenio. Phys. Rev. A, 57:1619, 1998.

[19] F. Verstraete, K. Audenaert, and B. De Moor. Phys. Rev. A, 64:012316, 2001.

[20] C.H. Bennett, D.P. DiVincenzo, J.A. Smolin, and W.K. Wootters Phys. Rev. A, 54:3824, (1996).

[21] R. Bhatia. Matrix Analysis, (Springer, 1997).

[22] W.J. Munro, K. Nemoto, and A.G. White. J. Mod. Opt., 48:1239 2001 . 